\title{
Effects of Mass Transfer between Martian satellites on Surface Geology
}

\author{
Michael Nayak ${ }^{1,2^{*}}$, Francis Nimmo ${ }^{1}$ and Bogdan Udrea ${ }^{3}$
}

\begin{abstract}
Impacts on planetary bodies can lead to both prompt secondary craters and projectiles that reimpact the target body or nearby companions after an extended period, producing so-called "sesquinary" craters. Here we examine sesquinary cratering on the moons of Mars. We model the impact that formed Voltaire, the largest crater on the surface of Deimos, and explore the orbital evolution of resulting high-velocity ejecta across 500 years using four-body physics and particle tracking.

The bulk of mass transfer to Phobos occurs in the first $10^{2}$ years after impact, while reaccretion of ejecta to Deimos is predicted to continue out to a $10^{4}$ year timescale (cf. Soter, Studies of the Terrestrial Planets, Cornell U., 1971). Relative orbital geometry between Phobos and Deimos plays a significant role; depending on the relative true longitude, mass transfer between the moons can change by a factor of five. Of the ejecta with a velocity range capable of reaching Phobos, $25-42 \%$ by mass reaccretes to Deimos and $12-21 \%$ impacts Phobos. Ejecta mass transferred to Mars is $<10 \%$.
\end{abstract}

We find that the characteristic impact velocity of sesquinaries on Deimos is an order of magnitude smaller than those of background (heliocentric) hypervelocity impactors and will likely result in different crater morphologies. The time-averaged flux of Deimos material to Phobos can be as high as $11 \%$ of the background (heliocentric) direct-to-Phobos impactor flux. This relatively minor contribution suggests that spectrally red terrain on Phobos (Murchie and Erard, Icarus 123, 63$86,1996)$ is not caused by Deimos material. However the high-velocity ejecta mass reaccreted to Deimos from a Voltaire-sized impact is comparable to the expected background mass accumulated on Deimos between Voltaire-size events. Considering that the high-velocity ejecta contains only $0.5 \%$ of the total mass sent into orbit, sesquinary ejecta from a Voltaire-sized impact could feasibly resurface large parts of the moon, erasing the previous geological record. Dating the surface of Deimos may be more challenging than previously suspected.

Icarus keywords:

Mars

Mars, satellites

1 Dept of Earth \& Planetary Sciences, Univ. of California at Santa Cruz. 1156 High St, Santa Cruz CA 95064.

2 Red Sky Research, LLC. 67 Northland Meadows Dr, Edgewood NM 87105.

3 Dept of Aerospace Engineering, Embry-Riddle Aeronautical Univ. 600 S Clyde Morris, Daytona Beach FL 32114.

*Corresponding Author (mnayak@ucsc.edu) 
Impact processes

Cratering

\section{Introduction}

Several features about the surface geology on the moons of Mars remain poorly understood. The grooves on Phobos, which do not exist on Deimos, have received the most attention (Horstman and Melosh, 1989; Thomas, 1979; Weidenschilling, 1979), and theories for their formation continue to be proposed to this day (Asphaug et al., 2015b; Basilevsky et al., 2014; Hamelin, 2011; Murray et al., 2006; Wilson and Head, 2015). However this is far from the only mystery. Though both moons are heavily cratered, with saturated surfaces and fine-grained regolith from impact debris accumulation (Lunine et al., 1982; Thomas, 1979), a large portion of ejecta produced on Deimos is retained in the form of crater fill of $\sim 5 \mathrm{~m}$ depth, a phenomenon not noted on Phobos (Thomas and Veverka, 1980a). This difference is still unexplained (Lee, 2009). The surface of Deimos is also significantly smoother and brighter than Phobos, likely a result of crater fill (Thomas, 1993; Thomas et al., 1996; Veverka, 1978).

Phobos also exhibits two distinct spectral units, one of "redder" origin and one of "bluer" origin, possibly stemming from a compositional difference (Lee, 2009; Murchie and Erard, 1996). The bluer unit is associated with the Stickney crater and an origin from depth. The redder unit associated with the surface and small craters; it is spectrally similar to D-type asteroids, but also to Deimos (Murchie and Erard, 1996, 1993). It has been proposed that the red unit is a wide-spread shallow layer superimposed on a blue base (Murchie and Erard, 1996), for which there are four possible causes (Britt and Pieters, 1988; Murchie et al., 1991): 1) accretion of Dasteroid material onto blue Phobos material; 2) optical alteration of the bluer unit; 3) accretion of ejecta from Martian basin impacts and subsequent space weathering or 4) Phobos is an inherently heterogeneous rubble pile and the red/blue units are end-member compositions. One aim of our study is to investigate the possibility that the red veneer on top of the base blue unit may be ejecta accreted from Deimos rather than Mars.

Previous work has established that impact ejecta can reimpact the target body or nearby companions after an extended period, creating so-called "sesquinary" impact morphology. Examples of sesquinary studies in the literature include Earth's Moon (Gladman et al., 1995), Io (Alvarellos et al., 2008), Ganymede (Alvarellos et al., 2002), Europa (Zahnle et al., 2008) and Pluto (Bierhaus and Dones, 2014). For Mars, previous work suggests ejecta released at slightly greater than the satellite's escape velocity could remain in the system and subsequently reimpact at low relative velocities (Soter, 1972,1971). Possible evidence for this was noted in analysis of Viking images (Veverka and Duxbury, 1977), however the efficiency of this process was previously unknown (Thomas, 1979). We report here on the distribution of impact velocities and geometries from inter-moon mass transfer trajectories, and 
present conclusions on the role and importance of sesquinary mass transfer between the Martian moons.

\section{Methods}

\subsection{Impact Model: Generating 2-D Velocity Streamlines}

Voltaire, the largest confirmed crater on Deimos, has a diameter of $3 \mathrm{~km}$ (Thomas and Veverka, 1980b; Thomas, 1979). By modeling the orbital evolution of ejecta from the Voltaire-forming impact, we aim to characterize an end-member case of mass transfer from Deimos to other Martian system bodies.

To model the streamlines ejected by the Voltaire impact, we use a simplified form of Maxwell's Z-model (Maxwell and Seifert, 1974; Maxwell, 1977; Roddy, 1977). Though limited by its neglect of interactions across streamlines, the Z-model reasonably approximates several experimentally observed features (Melosh, 1989; Richardson et al., 2007). The limitations of a Z-model implementation are discussed at length by (Barnhart and Nimmo, 2011). Our application is only concerned with ejecta streamlines that escape Deimos, and is unaffected by the details of cratering flow beneath the ground plane, surface material mixing during ejection or direct retention and emplacement of deposits. Therefore it provides a suitable level of insight into an outbound velocity distribution; approximations made by the Z-model are unlikely to alter our qualitative results.

We adopt the formulation of Barnhart and Nimmo (2011), who use $Z=2.71$ for a Mars application. When tested against numerical computations, $\mathrm{Z}=2.7$ represents surface explosion cratering flow well (Melosh, 1989). All streamlines are ejected at a constant angle of $35.4^{\circ}$ from the horizontal, set according to the relation (Maxwell, 1977):

$$
\varepsilon_{e j}=\tan ^{-1}(Z-2)
$$

Outbound radial $\left(v_{r}\right)$ and vertical $\left(v_{z}\right)$ ejection velocities vary inversely with distance from the center of the crater $r$ (Maxwell, 1977) as:

$$
\begin{aligned}
& v_{r}=\alpha / r^{Z} \\
& v_{z}=(Z-2) v_{r}
\end{aligned}
$$

where $g_{D}$ denotes the acceleration due to gravity for Deimos $\left(0.003 \mathrm{~m} / \mathrm{s}^{2}\right)$ and:

$$
\alpha=\sqrt{\frac{g_{D} R_{t}^{2 Z+1}}{4 Z(Z-2)}}
$$

Using a final crater radius $\mathrm{R}_{f}=1500 \mathrm{~m}$ for Voltaire (Veverka, 1978), the transient crater radius is calculated as $\mathrm{R}_{t}=0.65 \mathrm{R}_{f}$ (Barnhart and Nimmo, 2011). For the analysis presented here the number of streamlines $(n)$ has been chosen to yield a 
suitably dense streamline distribution with velocities greater than the Deimos escape velocity. Setting $R_{\text {min }}=0$ and varying $R_{\min } \leq \mathrm{r} \leq \mathrm{R}_{t}, n=600$ streamlines evenly spaced in radius are generated within the Voltaire crater. Converting streamlines into axisymmetric coordinates [cf. Barnhart and Nimmo, 2011, Fig 1], we extract the radial and vertical coordinates as:

$$
\begin{aligned}
& r=R_{i} \sin \theta(1-\cos \theta)^{\frac{1}{z-2}} \\
& z=R_{i} \cos \theta(1-\cos \theta)^{\frac{1}{z-2}}
\end{aligned}
$$

where $\theta$ is the angle from the vertical $\{\theta \mid \theta \in 0: \pi / 2\}$ and:

$$
R_{i}=\frac{R_{f}-R_{\min }}{n}
$$

\subsection{Creating 3-D velocity streamlines}

The 2-D axisymmetrical distribution is now used to create an approximation to a 3D excavation. The fate of the ejecta particle (reaccretion to Deimos, impact to Phobos, impact to Mars or escape) can vary greatly depending on the azimuth of the streamline. To rotate around the azimuthal direction, we define the Topocentric Horizon frame (Appendix A), adapted from the South-East-Zenith (SEZ) frame (Vallado, 2013). The azimuth of the ejection velocity vector $\beta$ is measured from the north, clockwise as viewed from above the impact site. We select a $30^{\circ}$ span as a compromise between computational efficiency and sampling a variety of azimuths across the possible solution space, such that $\beta \mid \beta \in(0: \pi / 6: 2 \pi)$ for a total of 11 possible azimuths. This yields a three-dimensional outbound velocity distribution tied to Voltaire. For use with the Mars gravity system integrator, these coordinates are then rotated into the Mars Centered Inertial (MCI) frame; details of coordinate transformations through the Deimos-Centered Deimos-Fixed (DCDF) and DeimosCentered Inertial (DCI) frames are presented in Appendix A.

Finally, we are specifically interested in those streamlines that have sufficient velocity to reach the orbit of Phobos. Since both moons lie in the same orbital plane (Cazenave et al., 1980), the minimum velocity at Deimos to reach Phobos can be analytically calculated with the Hohmann transfer [Section 6.3, Curtis, 2013]. Particles begin to cross the orbit of Phobos at velocities above $500 \mathrm{~m} / \mathrm{s}$, so we set the lower bound on velocities of interest at $400 \mathrm{~m} / \mathrm{s}$. From Deimos, the minimum velocity to escape the gravitational well of Mars is analytically approximated as [Eqn 2.80, Curtis, 2013]:

$$
v_{\text {esc }}=\sqrt{2 \mu / r_{\text {Deimos }}}
$$

where $r_{\text {Deimos }}$ is the distance from Deimos to Mars and $\mu$ is the product of the gravitational constant and the mass of Mars. From (8), $v_{\text {esc }}=1.91 \mathrm{~km} / \mathrm{s}$; we set the upper bound on velocities of interest at $2 \mathrm{~km} / \mathrm{s}$. Therefore, we examine velocity 
streamlines in the range $\{v \mid v \in 400: 2000 \mathrm{~m} / \mathrm{s}\}$. Nineteen of 600 streamlines fall within this range; rotated around 11 azimuthal positions, this creates a 209streamline distribution. While we focus here on ejecta with sufficient velocity to reach Phobos ( $\sim 400 \mathrm{~m} / \mathrm{s})$, we note that the majority of ejecta launched from Deimos at lesser velocities will ultimately re-impact Deimos.

\subsection{Planetary System Model}

Next we detail the formulation of the planetary model. Centered at the primary, the Mars gravity system is modeled with $12 \times 12$ gravity harmonics from the NASA Planetary Data System [pds-geosciences.wustl.edu] (Murchie, 2010). The effects of permanent solid tides are included, truncated to the size of the gravity field. The present-day orbit of Deimos is likely similar to its primordial orbit (Burns, 1978; Lambeck, 1979); we generate the 500-year orbits of Phobos and Deimos analytically from modern-day mean orbital parameters [ssd.jpl.nasa.gov, Table 1]. A subset of streamlines was run against high-precision orbits generated for Phobos and Deimos [Genova and Folkner, personal communication, 2015]; results were not found to differ substantially from those run against the analytical orbits. In the interest of computational speed, we adopt the analytical formulation hereafter.

Due to its proximity to Mars, the orbit of Deimos is primarily influenced by Mars' oblateness; the third-body effect from the Sun or other planetary bodies such as Jupiter is negligible (Burns, 1972). Ejecta released from the orbits of Deimos will follow a similar pattern; we therefore neglect these third-body effects. Similarly, solar radiation pressure is a second-order effect when compared to solar gravity perturbations (Farnocchia et al., 2014; Klacka, 2002); we neglect this effect as well. However for complete understanding of orbital evolution within the Martian system we include third-body perturbation effects from Phobos and Deimos, making the physics of our model a four-body problem.

Table 1. Mean Orbital Parameters and Constants for Phobos and Deimos

\begin{tabular}{|l|l|l|}
\hline & Deimos & Phobos \\
\hline Semi-major axis & $23485 \mathrm{~km}$ & $9389.8 \mathrm{~km}$ \\
\hline Eccentricity & 0.00115571 & 0.0164255 \\
\hline Inclination & $1.79 \mathrm{deg}$ & $1.09 \mathrm{deg}$ \\
\hline Right Ascension of Ascending Node & $148.0 \mathrm{deg}$ & $319.9 \mathrm{deg}$ \\
\hline Argument of Periapsis & $123.3 \mathrm{deg}$ & $270.7 \mathrm{deg}$ \\
\hline Mean longitude & $109.7 \mathrm{deg}$ & $190.6 \mathrm{deg}$ \\
\hline Rate of mean longitude & $0.00330049 \mathrm{deg} / \mathrm{s}$ & $0.0130317 \mathrm{deg} / \mathrm{s}$ \\
\hline Mean radius & $6.2 \mathrm{~km}$ & $11.3 \mathrm{~km}$ \\
\hline Acceleration due to gravity & $0.003 \mathrm{~m} / \mathrm{s}^{2}$ & $0.0057 \mathrm{~m} / \mathrm{s}^{2}$ \\
\hline Escape velocity & $5.56 \mathrm{~m} / \mathrm{s}$ & $11.39 \mathrm{~m} / \mathrm{s}$ \\
\hline
\end{tabular}

\footnotetext{
${ }^{1}$ Mean longitude is calculated with reference to a mean epoch coordinate system: The mean equator-mean equinox coordinate system is evaluated at the epoch of the object. The starting epoch is arbitrary due to our evaluation of the orbital dynamics at multiple relative geometry configurations between Mars, Phobos and Deimos that encompass all possible geometries between the bodies.
} 


\begin{tabular}{|l|l|l|}
\hline Hill sphere radius & $16.5 \mathrm{~km}$ & $21.5 \mathrm{~km}$ \\
\hline
\end{tabular}

We assume that any particle that enters the Hill sphere (Table 1) of either moon will be captured by it ${ }^{1}$. Due to its irregularly triaxial shape, Deimos has an uneven gravity field that causes the escape velocity to be lower at the sub-Mars and antiMars points [Davis et al., 1981]. Ejecta in the 4-6 m/s range will see the largest variation in range (Thomas, 1993); since the slowest particle we consider is ejected at $\geq 400 \mathrm{~m} / \mathrm{s}$ and the escape velocity varies on the order of $\mathrm{cm} / \mathrm{s}$, we can safely assume that the escape velocity at Voltaire equals the average escape speed over Deimos.

Finally we consider the impact of relative orbital geometry. Though an analytical formulation has been used to consider similar problems in the past (Dobrovolskis and Burns, 1980; Soter, 1971; Thomas, 1998), this approach may be insufficient for a full understanding of ejecta dynamics. Phobos is closer to Mars than any other planetary satellite, and is the only moon with an orbital period less than the rotational period of its primary body (Burns, 1972). The flux of material impacting Phobos can vary drastically between inferior and superior conjunctions between Phobos and Deimos. The difference in the true longitude between Deimos and Phobos can (and does, see Figure 1) change the outcome of a Phobos collision to a Mars collision, or vice versa. Therefore, though the orbits of Phobos and Deimos are generated analytically, all propagation in this work is ephemeris-centered.

Deimos has an orbital period of 30.3 hours, and Phobos 7.5 hours. To evaluate the fate of ejecta across the range of possible Mars-Deimos orbital geometries, we discretize this orbit into 28 geometry configurations (GCs), evenly spaced in onehour increments from the Deimos apoapsis. In this time period, Phobos completes nearly four orbits of Mars, allowing for discretization of the range of possible MarsPhobos-Deimos orbital geometries as well. At each GC, 209 streamlines are released and propagated, for a total of 5,852 streamlines, thereby ensuring a robust capture of the impacting process despite variations in orbital positions and conjunction geometries.

Estimates for lifetime of ejecta in the Martian system range from $10^{2}$ to $10^{4}$ years (Davis et al., 1981; Soter, 1971). We propagate each streamline in the Mars gravity system for $t_{\max }=500$ years, stopping sooner only in the event of a planetary body collision or departure from the Mars gravitational sphere of influence. This length of integration balances computational feasibility with permitting a statistically significant number of ejected particles to impact or escape. As we shall show, the uncertainty introduced by doing so does not affect our conclusions. It also permits the use of a Runge-Kutta integrator without excessive approximations to the

\footnotetext{
1 To test the validity of using the Hill sphere as an impact boundary, we selected 100 Phobos impact trajectories at random and integrated them with an impact boundary of $13 \mathrm{~km}$, the longest semimajor axis of Phobos (Murchie et al., 2003); 98/100 trajectories were still found to impact. Therefore, our qualitative results are not impacted by the use of the Hill sphere as an impact boundary.
} 
perturbed Hamiltonian (Leimkuhler and Reich, 2004). A seventh-order Runge-Kutta integrator with eighth-order error control is used for all orbit propagations. The maximum permitted relative error is $10^{-10}$. The Tisserand parameter is used to evaluate the performance of the integrator, according to which:

$$
\frac{1}{a}+2 \sqrt{a\left(1-e^{2}\right)} \cos i \cong \text { constant }
$$

where $a, e$ and $i$ are the semi-major axis, eccentricity and inclination of the orbit. The differences in the Tisserand parameter for an individual particle are no greater than $10^{-5}$, i.e., at most, a $0.001 \%$ change across the integration period.

\section{Results}

Figure 1 plots the fate of 5,852 massless ejecta particles in the Martian gravity system across 500 years as a function of the orbital geometry and Voltaire ejection velocity. The number of reaccretions to Deimos and particles still flying are relatively constant, with minor fluctuations. However, if the Voltaire impact occurs when Deimos is near periapsis, a spike in Mars impacts and a corresponding drop in particles escaping the system are noted. This is, in fact, due to the difference in true longitude between Phobos and Deimos at the time of ejecta launch; depending on the relative conjunctive geometry, the mass flux from Deimos to Phobos can be up to $500 \%$ higher.

It is surprising to note that there is more mass flux from Deimos to Phobos as opposed to Mars; intuitively, one would expect that most mass ejected from Deimos would either reaccrete or spiral down to Mars. Because greater mass is released at lower ejection velocities (O'Keefe and Ahrens, 1985), these results suggest impacts on Deimos may have an effect on Phobos' geology; we shall attempt to estimate the magnitude of that effect in Section 4. 

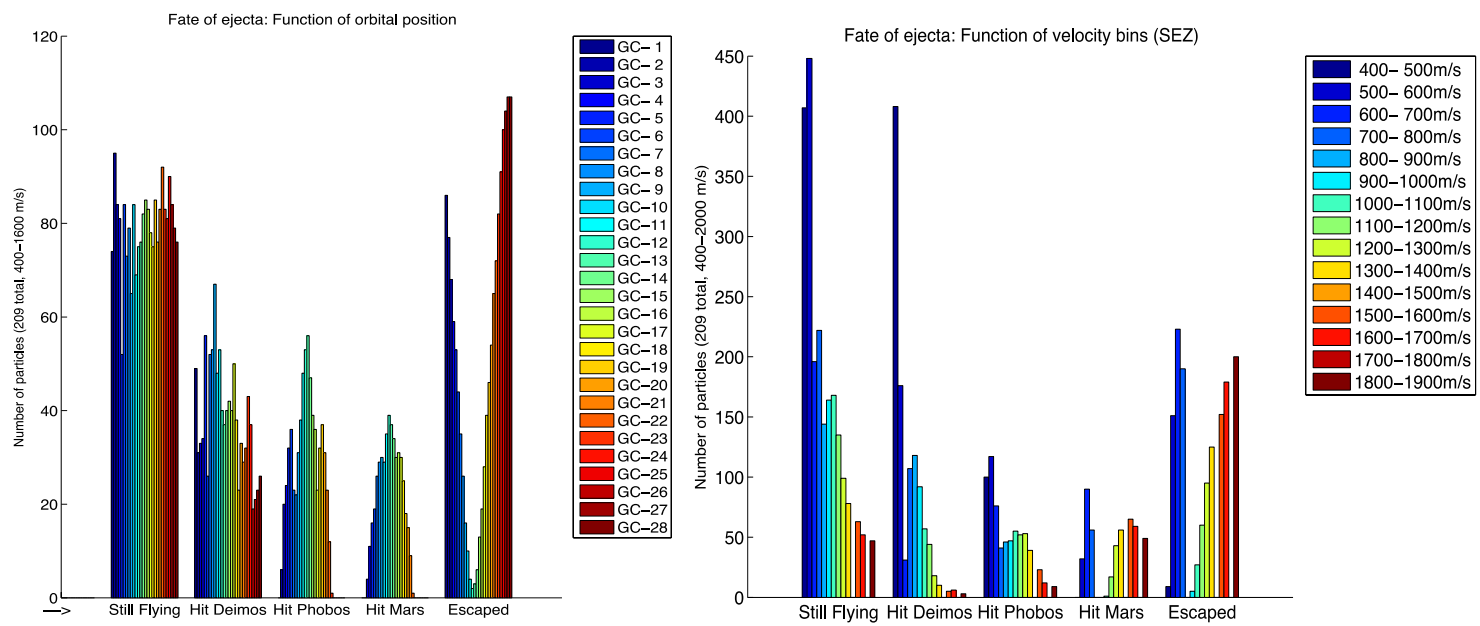

Figure 1. The fate of Deimos ejecta from Voltaire as a function of (Left) Orbital Geometry and (Right) Ejection Velocity from Voltaire. GC-1 is near-apoapsis; GC-14 is near-periapsis. Note that the difference in the Deimos-Phobos angle is the important quantity with regard to ejecta fate (see text).

For impacts with Mars, Phobos or Deimos, impact velocity is calculated with reference to the Planet-Centered Planet Fixed frame in question (Appendix A). We chart the variation of impact speeds at Phobos and Deimos across 28 GCs (Figure 2). For Phobos, regardless of orbital location at the time of ejecta release, impact velocity scales linearly with particle ejection velocity. Faster particles impact with higher speeds, in some cases up to $4 \mathrm{~km} / \mathrm{s}$ (though still not as high as $\sim 20 \mathrm{~km} / \mathrm{s}$ expected for heliocentric impactors). On Deimos, however, impacts above $1 \mathrm{~km} / \mathrm{s}$ are relatively rare. Almost no high-velocity $(>1 \mathrm{~km} / \mathrm{s})$ impacts are noted from nearapoapsis positions. $81 \%$ of impacts are clustered in the $0.4-0.8 \mathrm{~km} / \mathrm{s}$ region, implying that low-velocity reaccretions to Deimos are relatively common.
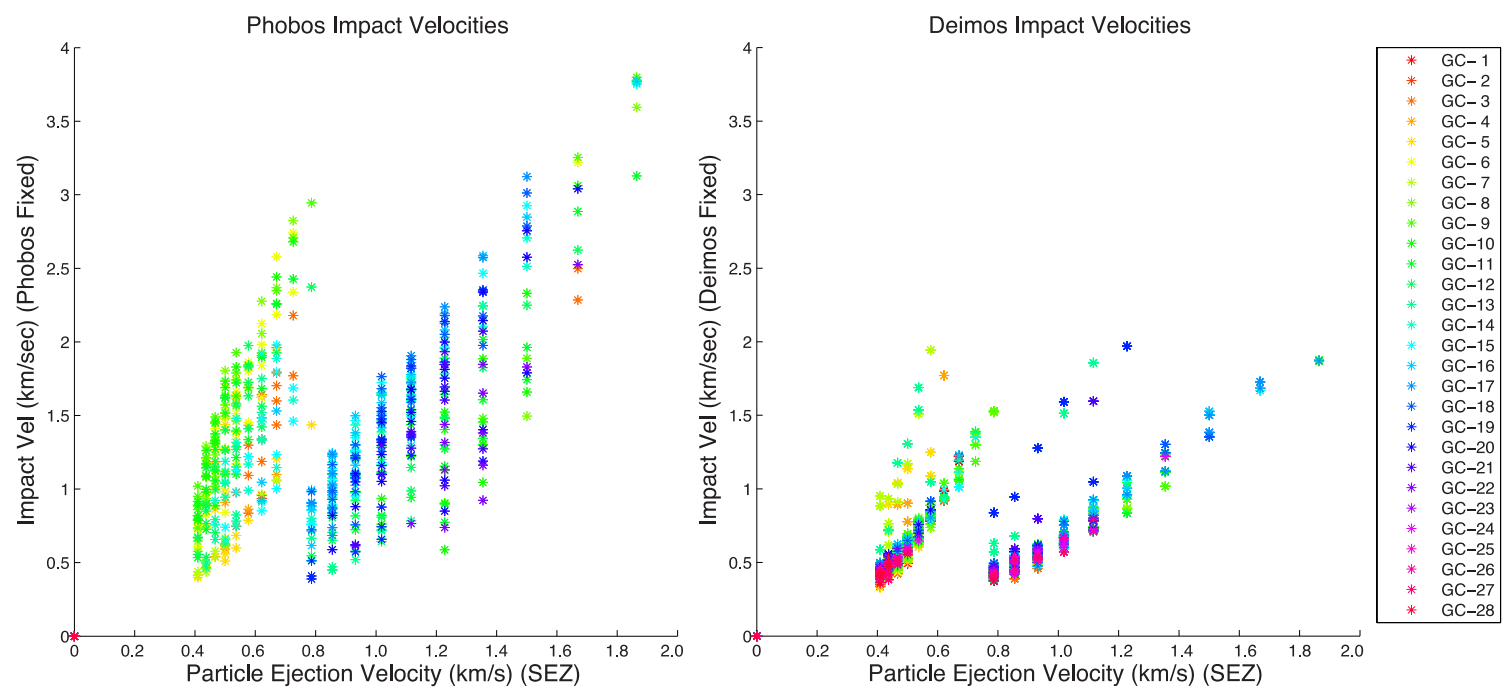

Figure 2. (Left) Impact Velocity at Phobos and (Right) Impact Velocity at Deimos versus particle ejection velocity from Voltaire. While Phobos exhibits several high-velocity impacts, high-velocity impacts at Deimos are relatively rare and are primarily clustered below $1 \mathrm{~km} / \mathrm{s}$ (compare to $\sim 20 \mathrm{~km} / \mathrm{s}$ heliocentric impactor velocity). The discontinuity at $700 \mathrm{~m} / \mathrm{s}$ in both graphs is due to an increase in impacts to Mars at that velocity range for certain Deimos-Phobos orbital geometries (Figure 1). 

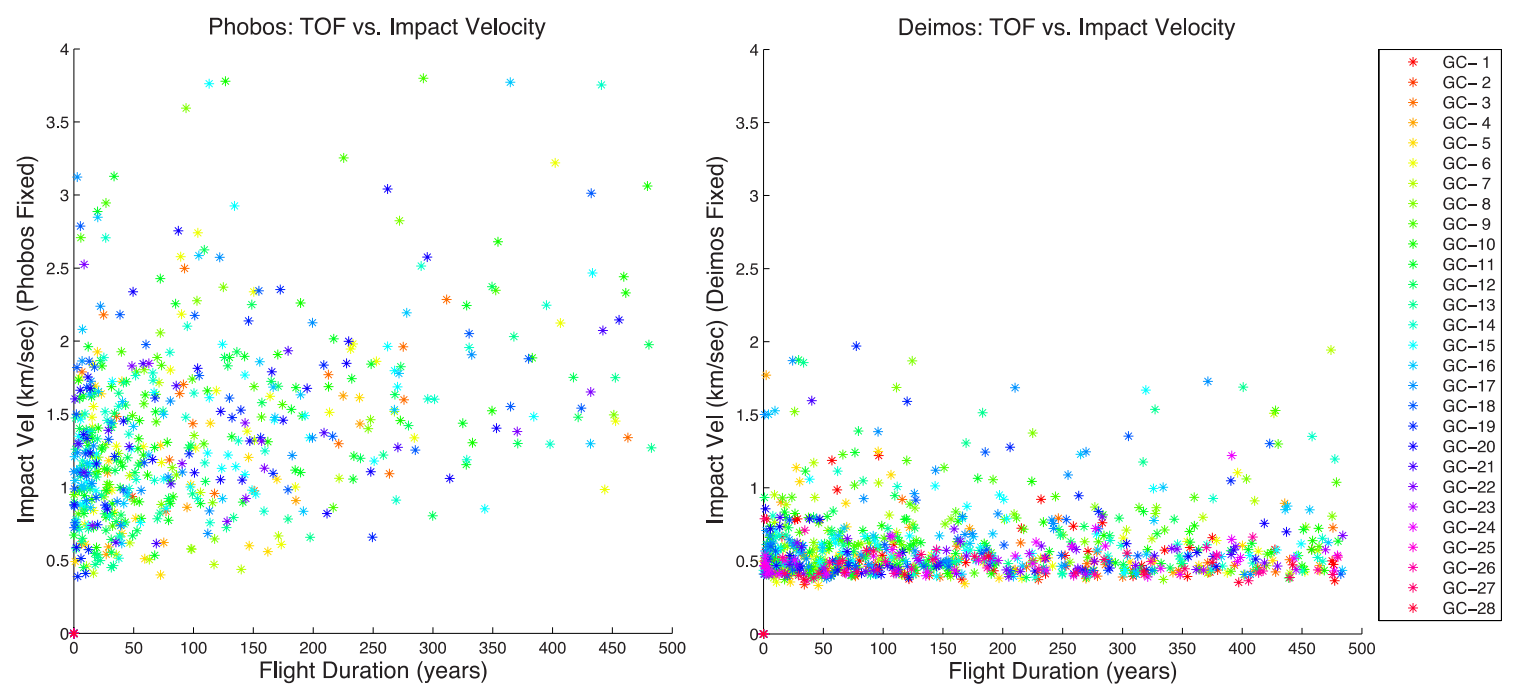

Figure 3. (Left) Impact velocity at Phobos and (Right) Impact velocity at Deimos versus duration of particle flight. While impacts to Phobos are frequent in the first 100 years post impact, they begin to taper off toward the end of the examined duration; impacts to Deimos, on the other hand, continue at a relatively constant pace, implying that while 500 years captures the bulk of Phobos mass transfer, reaccretions to Deimos will likely continue to the $\mathbf{1 0}^{4}$ year timescale (Davis et al., 1981; Soter, 1971).

Next, the relationship between impact velocities and the time to impact is examined. No significant acceleration effect with time or particular links to orbital geometry are noted (Figure 3). However, the contrast between the two bodies is again evident. On Phobos, the majority of impacts occur in less than 100 years; subsequent impacts become less frequent as time increases. This implies that the 500-year timescale selected is adequate to capture the majority of Deimos-to-Phobos material transfer. On Deimos, however, impacts continue to build, with the flux of impacts remaining relatively constant even at the end of the 500-year timescale. Therefore, it seems that while the majority of mass transfer to Phobos occurs early on, reaccretions to Deimos likely continue out to the $10^{4}$ year timescale hypothesized by Soter (1971). This also makes it likely that a large number of the particles still flying at the end of the 500-year simulation will end up reaccreting to Deimos. As a consequence, it is unlikely that increasing the computation time will qualitatively change our conclusions.

The flight path angle (FPA) is the angle between the incoming velocity vector and the position vector defined by the surface of the planet, and can be calculated as (Curtis, 2013):

$$
\cot \Upsilon=\frac{e \sin \Phi}{1+e \cos \Phi}
$$

where $e$ is the eccentricity of the impact trajectory and $\Phi$ is the true anomaly.

The relationship between impact velocity and FPA reinforces the rarity of highspeed reaccretion events on Deimos (Figure 4). On Phobos, the frequency distribution of impactor velocities and flight path angles suggests that impacts 
created by Deimos ejecta can vary from oblique, classically secondary impacts to direct cratering events.

Finally we test the fidelity of our results by investigating the likelihood of continuing collisions beyond the chosen 500-year timeframe. Figure 5 shows time curves for particles still flying and particles impacting Phobos or Deimos. As expected, Phobos impacts taper off with time, and the total number of Phobos impacts $(\mathrm{y})$ fits well $\left(\mathrm{R}^{2}\right.$ $>0.995)$ to a logarithmic distribution defined by $\mathrm{y}=178.4 \ln \left(\mathrm{t} / \mathrm{t}_{0}\right)$, where $t$ is time in years and the time constant $t_{0}$ is $\sim 10.46$ years. Deimos impacts continue to increase and fit well $\left(\mathrm{R}^{2}>0.995\right)$ to a distribution defined by $\mathrm{y}=469 \ln \left(\mathrm{t} / \mathrm{t}_{0}\right)$, where the time constant $t_{0}$ is $\sim 47.9$ years. Interestingly, the ratio between the time constants for Phobos and Deimos is similar to the ratio of their orbital periods.
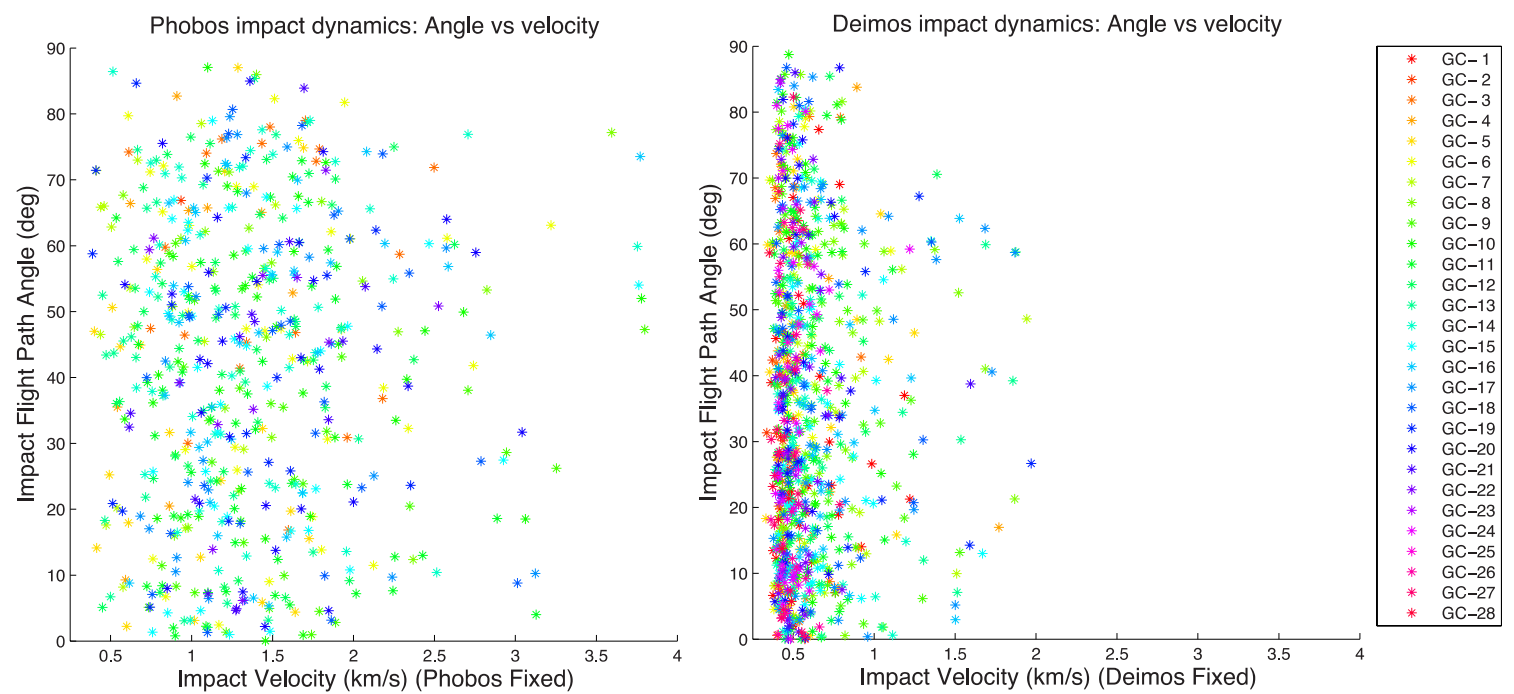

Figure 4. (Left) Impact FPA at Phobos and (Right) Impact FPA at Deimos versus the impact velocities on the respective bodies. Apart from reinforcing the fact that Deimos impacts are primarily low-velocity, we also see a wide distribution in flight path angles. There are several low-velocity, low-FPA impacts that should create oblique or secondary crater morphology, and several high-velocity, high-FPA impacts that will exhibit direct or primary crater morphology. 

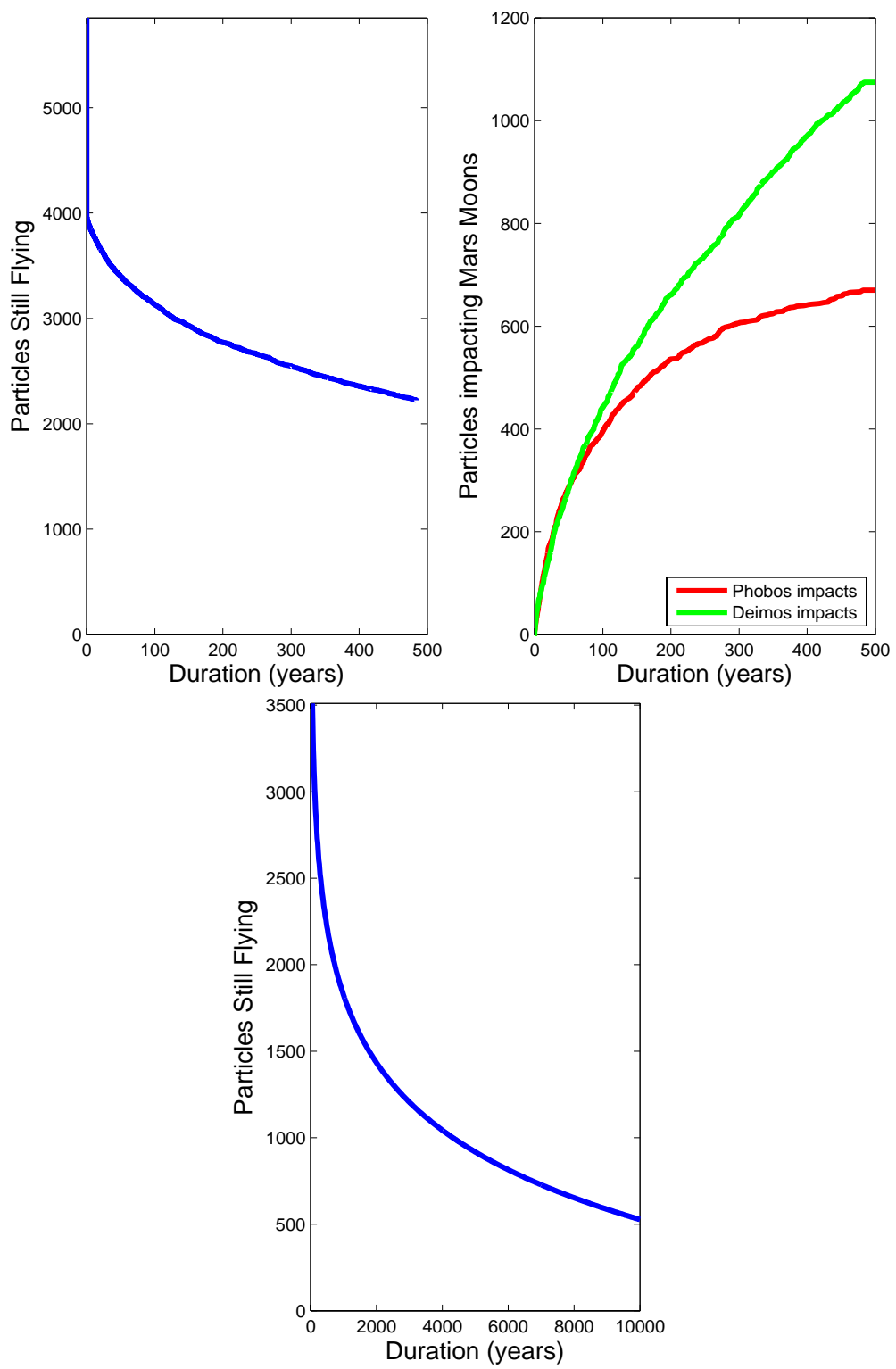

Figure 5. Number of (Left) particles still flying and (middle) impacts to Phobos and Deimos with time. The curves are well behaved, with no unexpected jumps. Impacts to Phobos can be seen to be tapering off, while reaccretions to Deimos continue to rise. These are expected to continue until no more particles are still flying. (Right) Extrapolation of the logarithmic decrease in the left plot. The decrease fits well $\left(R^{2}>0.995\right)$ to a logarithmic distribution defined by $y=-563 \ln \left(t / t_{0}\right)$, where $t_{0} \sim 25,000$ years. Using this curve, it takes $\mathbf{1 0 , 0 0 0}$ years for the particles still flying to drop below 500, agreeing with Soter (1971).

Reaccretions to Deimos are therefore expected to continue, but for how long? The shape of the graph for particles still in flight is in a logarithmic decrease; when extrapolated (Figure 5, right) it takes approximately 10,000 years for the number of particles still in flight to drop below $10 \%$ of the total number of particles generated. This result agrees well with predictions made by Soter (1971). However, a word of caution is appropriate here. Soter and this study both neglect effects of solar radiation pressure. While a second-order effect on the 500-year timescale, it can play a significant role across longer time periods, and could decrease the time to impact (Klacka, 2002). Based on this we do not expect our conclusions to change 
qualitatively with an increase in propagation time and corresponding decrease in number of particles still flying.

By analyzing the Martian system within the framework of an analytical restricted three-body problem, previous work finds that essentially all ejecta from either Phobos or Deimos will be reaccreted to the moon of origin (Dobrovolskis and Burns, 1980; Soter, 1971). Our differing results suggest that the four-body ephemerides formulation is critical to full understanding of the orbital dynamics.

\section{Mass Transfer between Martian Satellites}

To investigate the geologic impact of mass transfer between Phobos and Deimos, we need to convolve the probability distributions from Figure 1 with an appropriate mass-velocity distribution. Advanced scaling laws developed from numerical methods exist [e.g. Leinhardt and Stewart, 2012], but given the uncertainties associated with several key parameters we prefer a more transparent and simpler approach. For gravity-dominated cratering the volume ejected faster than a given velocity is (Holsapple, 1993; Housen and Holsapple, 2011):

$$
V=R^{3} C_{e j}\left(\frac{v_{e j}}{\sqrt{g R}}\right)^{-v}
$$

where $v_{e j}$ is the ejection velocity (from Figure 1 ) and $R$ is the final radius of Voltaire.

A sand-like surface is well represented by $v=1.2$ (Melosh, 1989). Experimental results that determine mass-velocity distributions for impacts into granular targets find $C_{e j}=0.25$ (Hermalyn and Schultz, 2013). Their results correlate well to the literature; Andrews (1975), Cintala et al (1999) and Stöffler et al. (1975) find $C_{e j}$ values between 0.25 and 0.36 . We adopt $C_{e j}=0.3$ and $v=1.2$. For Phobos' density we use $\rho=1.9$ g/cc (Avanesov et al., 1989; Rosenblatt et al., 2008; Schmedemann et al., 2014). From (11), the mass ejected within each velocity bin is calculated (Figure 6). In total, $3 \times 10^{9} \mathrm{~kg}$ is ejected from Deimos between $400-2000 \mathrm{~m} / \mathrm{s}$. 

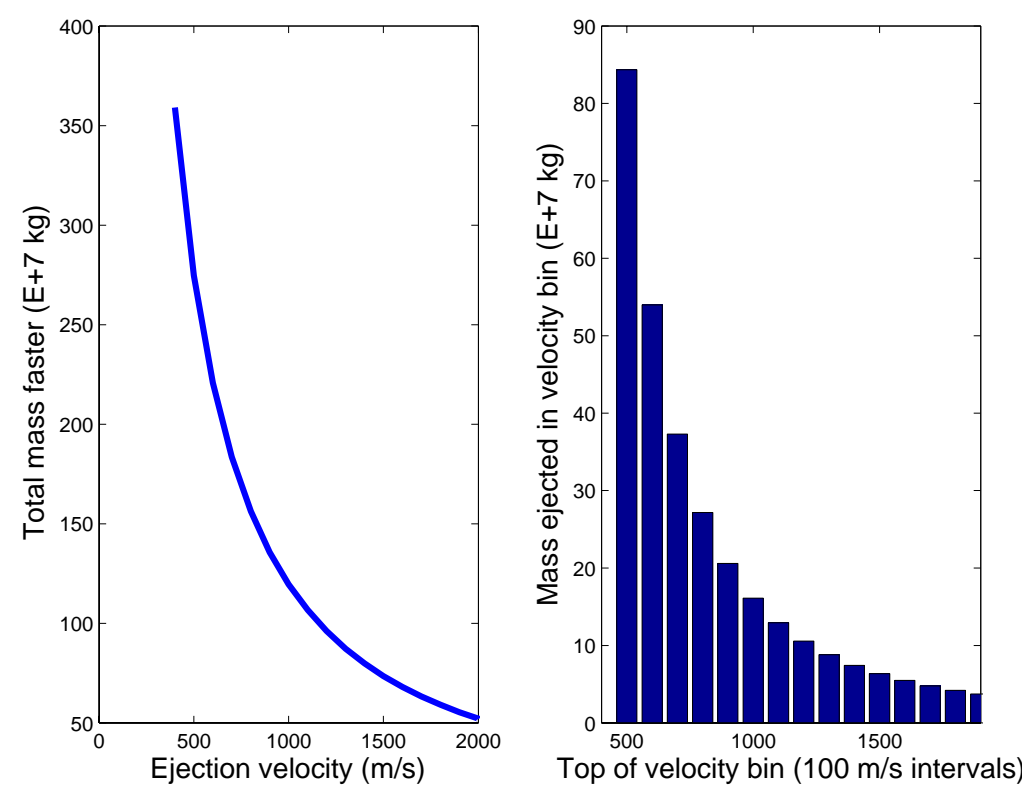

Figure 6. (Left) Total mass faster than ejection velocities (Equation 11); (Right) Mass ejected per velocity bin in tens of millions of kg. Velocity bins correspond to Figure 1.

Using (11) and the acceleration due to gravity of Deimos $\left(0.003 \mathrm{~ms}^{2}\right)$, the total mass excavated faster than escape velocity is $6.1 \times 10^{11} \mathrm{~kg} ; 0.5 \%$ of this total is therefore ejected in the $400-2000 \mathrm{~m} / \mathrm{s}$ velocity range. Of this $0.5 \%$, what percentage reaches Phobos? From integrating the mass delivered per velocity bin (Figure 6) and averaging it across impacts at all GCs (Figure 1), approximately $3.5 \times 10^{8} \mathrm{~kg}$ impacts Phobos. This is $12 \%$ of the total mass released in the $400-2000 \mathrm{~m} / \mathrm{s}$ range. This is also $21 \%$ of the mass not still in orbit at the end of the simulation ("still flying", Figure 1). If all this mass were ultimately to impact Phobos, the total impacting mas would be $6 \times 10^{8} \mathrm{~kg}$, the likely maximum value. Therefore, $12-21 \%$ of the mass that can reach Phobos does end up on Phobos on a 104-year timescale.

The same analysis yields $7.2 \times 10^{8} \mathrm{~kg}$ impacting Deimos, which is $25 \%$ of the total mass released in the $400-2000 \mathrm{~m} / \mathrm{s}$ range and $42 \%$ of the mass in this range not still flying. This yields an upper bound of $1.2 \times 10^{9} \mathrm{~kg}$ impacting Deimos. Ejecta launched at lower velocities than $400 \mathrm{~m} / \mathrm{s}$ cannot reach Phobos and will mostly re-impact Deimos. Mars only receives 5-9\% of ejecta in the 400-2000 m/s range. The remaining ejecta escapes the Mars system into heliocentric space.

\subsection{Comparison with Background Flux}

We seek to place the sesquinary mass flux into perspective by comparing it to the estimated background mass flux from meteoroidal impacts. The exact flux of small meteoritic bodies at Mars orbit is not known, so we derive a formulation dependent on a characteristic timescale that eliminates the Mars mass flux quantity. Brown et al (2002) use data from geostationary satellites around the Earth to estimate a 
power law relationship between the number of objects colliding with the Earth per year $(N)$ with diameters of at least $D$, of the form:

$$
\log N=c_{0}-d_{0} \log D
$$

where $c_{0}=1.568 \pm 0.03, d_{0}=2.70 \pm 0.08$. Assuming the same power law distribution for Martian system bodies, for $N=N_{\text {planet }}$ this can be reformulated as:

$$
N_{\text {planet }}(d>D)=C_{\text {planet }} D^{-d_{0}}
$$

where $C_{\text {planet }}=10^{C_{0}}$. As we will show, the value of this constant does not matter for our analysis. Assuming spherical impactors with diameter $D$ and density $\rho_{i}$, the incremental number of impactors $d N$ per year results in a mass flux increment of

$$
d M=\frac{1}{6} \rho_{i} \pi D^{3} d N
$$

kg per year. Differentiating (13), substituting into (14) and integrating to $D_{\max }=D$, the mass accumulated by a generic planetary body in $\mathrm{kg} / \mathrm{yr}$ from asteroidal flux is:

$$
M_{\text {planet }}(d>D)=C_{\text {planet }}\left[\frac{d_{0}}{3-d_{0}}\right]\left[\frac{\pi \rho_{i}}{6}\right] D^{3-d_{0}}
$$

where $d_{0}<3$. Zahnle et al (2003) derive a relationship for the impact rate of a satellite compared to its planet. Applied to the Mars system, this is:

$$
N_{P h o}(d>D)=N_{\text {mars }} f_{\text {Pho }}
$$

where $D$ is the diameter of the largest impactor incident to Phobos and:

$$
f_{P h o}=\frac{R_{P h o}^{2}}{R_{\text {mars }} a_{P h o}}
$$

where $a$ is the distance from Mars. The resulting values are $5 \times 10^{-7}$ and $4 \times 10^{-6}$ for Deimos and Phobos respectively. Combining (13) and (16) for Phobos:

$$
C_{P h o}=\frac{f_{P h o}}{D_{s t}^{-d_{0}}} N_{\text {mars }}^{s t}
$$

where $N_{\text {mars }}^{s t}$ signifies $N_{\text {mars }}\left(d>D_{s t}\right)$. The assumption here is that Stickney is the largest impact to have occurred on Phobos over its history and the diameter of the Stickney-forming impactor is $D_{s t}$. Substituting (18) into (15), and applying (13) to $N_{\text {mars }}^{\text {st }}$ :

$$
M_{P h o}\left(d>D_{s t}\right)=C_{m a r s} f_{P h o}\left[\frac{d_{0}}{3-d_{0}}\right]\left[\frac{\pi \rho_{i}}{6}\right] D_{s t}^{3-d_{0}}
$$


Next, we define a characteristic timescale $\tau$ defined such that $\tau$ years elapse between Voltaire-size collisions on Deimos. From (16) and the definition of $N$ :

$$
\tau=1 / N_{\text {Dei }}=1 /\left(f_{\text {Dei }} N_{\text {mars }}^{\text {vol }}\right)
$$

where $N_{\text {mars }}^{\text {vol }}$ is $N_{\text {mars }}\left(d>D_{\text {voltaire }}\right)$ for Deimos. Expanding this according to (13):

$$
\tau=1 /\left(f_{\text {Dei }} C_{\text {mars }} D_{\text {vol }}^{-d_{0}}\right)
$$

Across $\tau$ years, combining (19) and (21), the poorly known (and, on long timescales, time-variable) annual mass flux delivered to Mars represented by $C_{\text {mars }}$ cancels. The total mass accreted by Phobos due to background impacts on a Voltaire timescale is:

$$
m_{P h o, a c c}=\tau M_{P h o}=\frac{f_{P h o}}{f_{D e i}}\left[\frac{d_{0}}{3-d_{0}}\right]\left[\frac{\pi \rho_{i}}{6}\right] \frac{D_{s t}^{3-d_{0}}}{D_{v o l}^{-d_{0}}}
$$

Equation (22) is only dependent on the diameter of the impactors and the slope of the size-frequency distribution. For Phobos, the largest crater is Stickney, with a 170-m likely impactor size (Asphaug and Melosh, 1993). For Deimos, the Voltaire impactor diameter is estimated from gravity-dominated scaling relations, rearranged from Cintala and Grieve (1998) and Schmidt and Housen (1987) and similar to Zahnle et al (2003):

$$
D_{v o l}=\left(0.862 D_{t}\left(\frac{\rho_{D}}{\rho_{i}}\right)^{\frac{1}{3}} V_{i}^{-0.44} g_{D}^{0.22}\right)^{1.2821}
$$

where the subscript $i$ denotes the impactor that created Voltaire, subscript $D$ denotes Deimos, units are CGS and $D_{t}$ is the diameter of the Voltaire transient crater, taken to be $1.95 \mathrm{~km}$. Asphaug and Melosh (1993) assume an impact of $3 \mathrm{~km} / \mathrm{s}$ for the Stickney impact; assuming the same impact velocity and impactor density, (23) yields a Voltaire impactor diameter of $25 \mathrm{~m}$. From (22), for an asteroid-type impact $\left(\rho_{i}=2.6 \mathrm{~g} / \mathrm{cc}\right.$; Barnhart and Nimmo (2011)), the mass accreted by Phobos from solar system impactors between Voltaire-scale impacts is approximately $2.9 \times 10^{9} \mathrm{~kg}$; it would be less if the impactor were assumed to have the lower characteristic density of Phobos. Comparing this to the $3.5 \times 10^{8} \mathrm{~kg}$ that impacts Phobos during every Voltaire impact, the fraction of Deimos material delivered to Phobos $(F)$ represents, on average, 0.12 of the material accreted to Phobos from direct solar system impactor flux.

Using a similar derivation for Deimos, we find that only $2 \times 10^{8} \mathrm{~kg}$ is accreted to Deimos by solar system impactors between Voltaire-size events. Deimos receives less material than Phobos because the focusing factor (Equation 17) is smaller. This flux is exceeded greatly by the reaccreted mass ejected during a Voltaire-size impact; for ejecta with velocities $400-2000 \mathrm{~m} / \mathrm{s}, F=3.6$. However this velocity range is only $0.5 \%$ of the total mass thrown into orbit. Ejecta with velocity $<400 \mathrm{~m} / \mathrm{s}$ has 
insufficient velocity to reach Phobos (or Mars) and will likely reaccrete to Deimos. Thus, the true value of $F$ across all ejecta is likely $\sim 700$ for Deimos. In other words, on Deimos, mass reaccreted during a Voltaire-size impact greatly exceeds mass naturally accreted from solar system impactors. However, sesquinaries from a large impact on Deimos provide only a minor contribution to the flux at Phobos.

As a reality check, we calculate the applicability of (12) to Mars system impacts. The frequency of a Voltaire-sized impact is $\tau=1 / N_{\text {dei }}$ years, or $134 \mathrm{Ma}$. We discuss the issue of the apparent age of Voltaire further below. Using (21), the ratio of the timescale for a Voltaire-forming impact on Deimos to a Stickney-forming impact on Phobos is 0.049 . Therefore, the frequency of a Stickney-sized impact is approximately every $2.7 \mathrm{Ga}$, which seems quite reasonable.

As an additional check, we estimate the mass flux to Mars from studies in the literature. Chappaz et al (2011) find that the mass flux from Mars to Phobos can be estimated at $0.25 \mu \mathrm{g} / \mathrm{m}^{3} / \mathrm{yr}$ or $0.2 \mathrm{~kg} / \mathrm{yr}$ across the inner moon's surface area assuming a mixing depth of $0.5 \mathrm{~m}$. The mass flux of solar system projectiles to Phobos is numerically found to be $k=40-2400$ times greater than the flux from Mars ejecta, with a Monte Carlo preferred value of $k=195$ (Ramsley and Head, 2013). The preferred value yields a mass flux at Phobos of $38.5 \mathrm{~kg} / \mathrm{yr}$ from asteroids, comets and meteoroids. At this rate, across $134 \mathrm{Ma}$, Phobos accumulates $5.2 \times 10^{9} \mathrm{~kg}$ from direct impacts, within a factor of two of the $2.9 \times 10^{9} \mathrm{~kg}$ derived above.

While independent of Mars' meteoroidal flux, the results are admittedly susceptible to the assumed size of the Voltaire impactor. The size of Stickney implies that the gravity regime approximation is likely appropriate. However the short timescale derived for the Voltaire impact implies that it may be deeper in the strength regime than initially assumed. If true, the Voltaire impactor would have to be larger than 25 $\mathrm{m}$, which would reduce quantitative estimates of $F$, the fraction of Deimos' mass flux with respect to the solar system impactor mass flux.

Assuming that the impactor was incident at a $45^{\circ}$ angle (Holsapple, 1993; Melosh, 1989), we can estimate the diameter of an impactor for strength-dominated cratering by rearranging Equation (12) from Zahnle et al (2008) to yield:

$$
D_{v o l}=1.027\left(\frac{Y}{V_{i}^{2}} \frac{D_{t}^{3}}{\rho_{i}}\right)^{\frac{1}{3}}
$$

where $Y$ represents the dynamic strength of the body; other variables are as in (23). Melosh (1989) uses a value of $Y=2 \mathrm{MPa}$, the observed yield stress at crater collapse, to estimate the gravity/strength transition on the Earth and the Moon. Adopting this value in (24), the diameter of the Voltaire impactor is $88 \mathrm{~m}$. However, the value of $Y$ is uncertain; for the low-density Deimos, it is unlikely that the yield strength is as high as $2 \mathrm{MPa}$. For an order of magnitude change in $Y$ ranging from 0.2 to $20 \mathrm{MPa}$, the diameter of the impactor varies from 40 to $190 \mathrm{~m}$. 
Is strength or gravity scaling more appropriate for modeling the Voltaire impact? Gravity can be a factor on solar system bodies as small as $400 \mathrm{~m}$ (Love and Ahrens, 1996). Phobos and Deimos have similar compositions, bulk densities and accelerations due to gravity (Davis et al., 1981; Szeto, 1983), and several arguments for modeling cratering on Phobos in the gravity-dominated regime are detailed in Asphaug and Melosh (1993) and Asphaug et al (2015a). Finally, while some authors have used the wide distribution of ejecta on Deimos to surmise that strength-scaling may be appropriate for Deimos (Lee et al., 1986), we suggest that this global distribution could instead be a function of the large amount of mass reaccreted over $500 y r$ timescales (Section 3). However, even if the Voltaire impact is in the strength regime, this would not change our qualitative results, i.e., 1) that the sesquinary mass transfer is a relatively small fraction of meteoroidal impacts to Phobos, and 2) that the mass reaccreted to Deimos from large Deimos impacts exceeds the meteoroidal mass flux to Deimos. For instance, increasing the diameter of the Voltaire impactor to $88 \mathrm{~m}$, the qualitative results become $F=0.004$ for Phobos and $F$ $=24$ for Deimos across all ejecta velocities. Therefore, uncertainty on where the Voltaire impact lies in the gravity/strength regime does not affect our qualitative conclusions.

\section{Discussion}

\subsection{Importance of Inter-moon Mass Transfer and Re-accretion}

The central result of this work concerns the relative mass transfer during impacts on Deimos. We have found that a Voltaire-sized impact on Deimos does transfer mass from Deimos to Phobos, with sufficient velocity to create primary crater morphology, discussed further below. When viewed with reference to the solar system impactor flux, the sesquinary mass transfer is not significant, and is likely in the $10 \%$ range. However, compared to the 25 ppm of Mars material in Phobos regolith estimated by Chappaz et al (2011), one out of ten particles originating from Deimos is a large number and is of interest to Phobos lander mission concepts [e.g. Udrea et al., 2015, 2016].

While the placement of Voltaire within the strength-gravity domain can cause some uncertainty in impactor size, we have shown that the total ejecta mass reaccreted to Deimos is likely to exceed the background mass flux to Deimos $(F>20)$ greatly. A Voltaire-sized impact could therefore feasibly resurface large parts of the moon, erasing the previous geological record. Dating the surface of Deimos may be more challenging than previously suspected; the surface age may better represent the age of either Voltaire or the similarly sized Swift crater. Further, an 11-km concavity on the southern end of Deimos is hypothesized to be a possible impact scar from an ancient, very large collision (Lee et al., 1986; Thomas, 1993; Thomas et al., 1996). If true, this would have resulted in the transfer of significant sesquinary mass transfer to Phobos, and a complete resurfacing of Deimos's surface. 


\subsection{The Spectral Dichotomy of Phobos}

Phobos exhibits two distinct spectral units, one of "redder" origin and one of "bluer" origin, a distinction that likely stems from a compositional difference (Lee, 2009; Rivkin et al., 2002). It has been proposed that the red unit is a wide-spread shallow layer superimposed on a blue base that is perhaps more representative of Phobos composition at depth (Murchie and Erard, 1996; Murchie et al., 2008). Possible mechanisms for the superimposition of red material are briefly outlined in Section 1 (Britt and Pieters, 1988). These include the hypothesis that the spectrally red "veneer" may be ejecta accreted from Deimos rather than Mars, as suggested by, e.g., Smith et al (2015).

Our results for the distribution of low-velocity, oblique-angle impacts (Figure 4) support the existence of trajectories that could, in theory, deposit a "veneer" of red Deimos material across Phobos's surface. However, the inter-moon mass flux is relatively small compared to the solar system impactor flux, which likely has a greater effect on the global surface geology, particularly in the 100+ Ma since the last Voltaire-sized impact. Therefore we believe it unlikely that the red veneer of Phobos is of Deimos origin. Recent spectral analysis by Thomas et al. (2011) supports this finding with evidence of subsequent impacts penetrating the blue unit near Stickney to reveal redder material. This observation casts doubt on the idea that the blue unit may be representative of Phobos at depth (Basilevsky et al., 2014). Ultimately, sample return from both Phobos and Deimos will conclusively establish which spectral unit is representative of depth; our results suggest that the surface unit is unlikely to originate from Deimos.

\subsection{Primary versus Secondary Impact Morphology}

Due to the dearth of classic secondary impact features such as radial crater chains or herringbones, it has been concluded that few, if any Phobos craters are secondary in origin (Thomas and Veverka, 1980b; Thomas, 1979). A similar conclusion was reached for Deimos. A limit of $10 \mathrm{~m} / \mathrm{s}$ on maximum re-impact velocity was proposed by Thomas (1998) and continues to be used in analysis of Phobos' geology (Murray et al., 2006; Schmedemann et al., 2014; Smith et al., 2015). However, every sesquinary impact studied here occurs at speeds above $100 \mathrm{~m} / \mathrm{s}$. Though still orders of magnitude below heliocentric impactor velocities, Figure 4 shows several highvelocity, high-FPA particles that could create craters indistinguishable from primary impact craters on Deimos, and particularly on Phobos.

On Deimos, over $80 \%$ of impacts cluster in the $0.6 \pm 0.2 \mathrm{~km} / \mathrm{s}$ region, implying that the majority of reaccretions are low velocity (subsonic). However for Phobos sesquinary particles can arrive with either subsonic or supersonic impact velocities. One would therefore predict a wide range in the resulting crater morphology; a comprehensive image survey of Phobos may be able to distinguish between the different crater morphologies. We find several low-velocity, low-FPA particles 
incident to Phobos that could create classic oblique or chained secondary impact morphology; a Phobos image survey by Smith et al (2015) finds several craters and deposits likely originating from such low-velocity impacts.

The escape speed from Mars at Phobos' orbit is $3 \mathrm{~km} / \mathrm{s}$, which is exceeded by the fastest sesquinary impacts (Figure 2). These impacts can create ejecta of their own that may subsequently be lost from the Mars system. Mass loss from Phobos is one possible explanation for why outlines of ejecta blankets are not conspicuous on Phobos (Lee et al., 1986). Additional simulations of Phobos-centered ejecta dynamics are needed to confirm this, and are planned as future work.

\subsection{Deimos Surface Smoothness, Brightness and Sesquinary Impact Gardening}

Very little ejecta escapes large bodies (e.g. Earth), with the majority redeposited locally as a continuous ejecta blanket. Most ejecta escape from very small bodies (e.g. Phoebe (Burns et al., 1996)), never to be reaccreted. Ejecta dynamics on Deimos present an interesting bridge between these two regimes, with sesquinary effects appearing to be important. Ejecta escapes but is then reaccreted on a timescale of up to hundreds of years, resulting in a global, near-isotropic redistribution of sesquinary ejecta.

For a Voltaire-sized impact, almost all of the ejecta material launched at velocities < $400 \mathrm{~m} / \mathrm{s}$ will ultimately reaccrete to Deimos (Soter, 1972; Thomas et al., 1996). From equation (11) above this represents $6.1 \times 10^{11} \mathrm{~kg}$, or about $0.5 \mathrm{~m}$ thickness of material distributed evenly over the entire surface. We estimate that the Voltaire and Swift impacts together could have added on the order of $1 \mathrm{~m}$ of fresh regolith or crater fill to Deimos. It has been suggested that the smoother and brighter surface of Deimos is due to crater fill of 5-7 m depth (Thomas, 1979, 1993; Thomas et al., 1996; Veverka, 1978). Given our estimate, we suggest that reaccreting sesquinary mass provides at least a partial explanation for the origin of this crater fill material.

The impact velocity distributions (Figure 2) also suggest a tertiary ejecta effect. Though most sesquinary impacts to Deimos are relatively low speed $(<1 \mathrm{~km} / \mathrm{s})$, these are still significantly higher than the escape velocity of Deimos $(5.5 \mathrm{~m} / \mathrm{s}$, Table 1) and could potentially launch additional ejecta in their turn (see below). Similarly, ejecta from Deimos can impact Phobos with enough mass and speed to create craters and excavate Phobos mass, which could then enter Mars orbit.

On Deimos, sesquinary impacts represent a large mass flux relative to the background flux and have $\sim \mathrm{km} / \mathrm{s}$ impact velocities (Figure 2 ). The result is likely to be the production of further suborbital, ballistically emplaced ejecta. This mechanism could contribute to the smooth appearance of the Deimos surface. Energy and momentum transfer from reaccretion impacts might even set off downslope movement noted on Deimos (Thomas and Veverka, 1980a), though 
admittedly the efficiency of the impacts at initiating this process is unknown and should be constrained in the future.

The possibility of sesquinary impact gardening makes the evolution of regolith in between major collision events a complex process on both Martian moons.

Determining the exact nature of Deimos material mixed with Phobos regolith is one of the primary science objectives of lander concepts in development for Phobos (Udrea et al., 2016, 2015). The methods applied in this work are further applicable to understanding regolith development and dust belts on small bodies within planetary gravitational wells, such as Phoebe and Iapetus in the Saturnian system.

\section{Acknowledgements}

This research was conducted with Government support under and awarded by the Department of Defense, National Defense Science and Engineering Graduate (NDSEG) Fellowship, 32 CFR 168a, with supplementary support to M.N. provided by Red Sky Research, LLC. We gratefully acknowledge computational support provided by Elizabeth Hyde, as well as the NASA Ames Research Center through a contract with the University of California Santa Cruz University Affiliated Research Center (UARC). Thanks to Bill Folkner (JPL/Caltech) for high-fidelity long-term Phobos/Deimos SPICE orbit propagations, and to Anthony R. Dobrovolskis and an anonymous reviewer for insightful comments that strengthened this manuscript. This research made use of NASA's HORIZONS (ssd.jpl.nasa.gov), NAIF (naif.jpl.nasa.gov) and PDS (pds.nasa.gov) databases. Opinions, interpretations and recommendations expressed are those of the authors and are not necessarily endorsed by the US Air Force or the Department of Defense.

\section{Appendix A: Coordinate Transformations}

This section details coordinate transformations necessary to use surface-centered Zmodel streamlines in the Mars-centered simulation. Three planet-based frames are used and detailed here.

\section{A.1 Planet-Centered Inertial (PCI) frame}

The origin of the PCI frame is the center of the planet: Mars, Deimos or Phobos. The positive $\mathrm{x}$-axis points toward the vernal equinox, the positive $\mathrm{z}$-axis extends through the North Pole of the planet and the y-axis completes the right hand system. In this definition, the North Pole is that pole of rotation that lies on the north side of the invariable plane of the solar system (Archinal et al., 2010). The planetary system model described in Section 2.3 is placed with reference to the Mars Centered Inertial (MCI) frame. 


\section{A.2 Planet-Centered Planet-Fixed (PCPF) frame}

Like the PCI frame, the origin of the PCPF frame is also the center of the planet, and shares its z-axis definition. However, the $\mathrm{x}$-axis extends through the intersection between the planet's equator and its prime meridian, with the $y$-axis completing the right hand system.

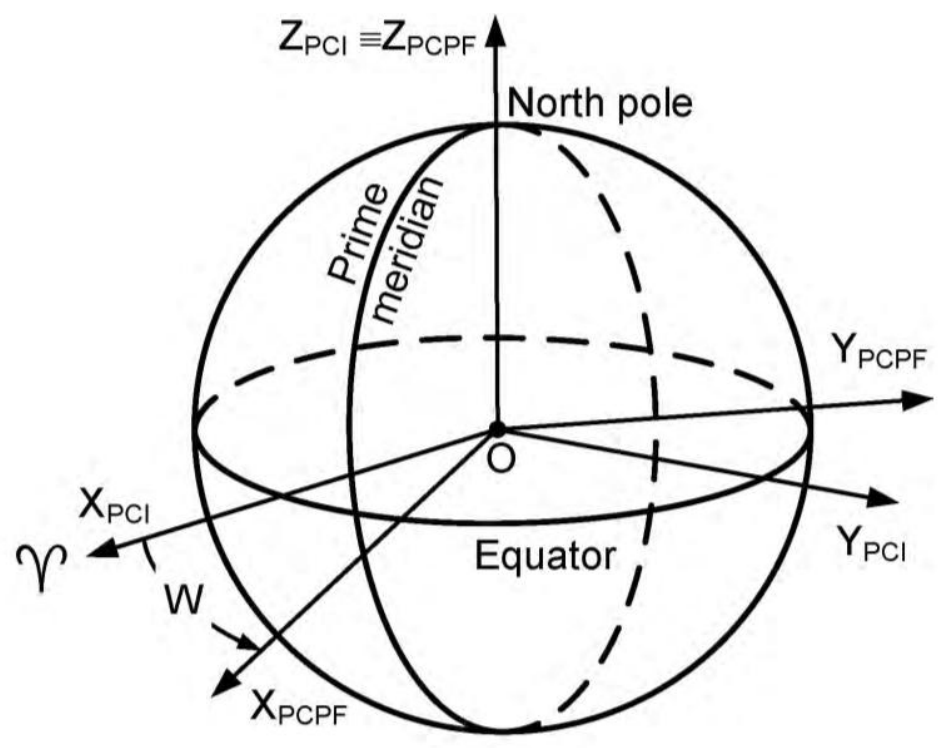

Figure 7. Illustration of the relationship between the Planet-Centered Inertial (PCI) and Planet-Centered Planet-Fixed (PCPF) frames

Figure 7 illustrates the relationship between the PCI and PCPF frames. PCI can be rotated into the PCPF frame around the z-axis with the rotation matrix:

$$
{ }^{P C P F} R^{P C I}=\left[\begin{array}{ccc}
\cos w & \sin w & 0 \\
-\sin w & \cos w & 0 \\
0 & 0 & 1
\end{array}\right]
$$

\section{A.3 Topocentric Horizon frame (also SEZ: South-East-Zenith frame)}

The topocentric horizon frame is adapted from the South-East-Zenith (SEZ) frame as defined by Vallado (2013); we refer to the two interchangeably here. The definition of the Topocentric Horizontal frame assumes a sphere centered at the center of mass of Deimos and tangent to the origin of the frame, which is the center of the impact site (Voltaire). The $\mathrm{x}$-axis is aligned with the meridian that passes through the center of Voltaire and points south. The $y$-axis is defined such that the $x$-y plane is tangent to the surface of Deimos at the center of Voltaire and points along the local latitude circle. Completing the right-handed system, the z-axis points radially outward from the impact site towards the "local" zenith. The local horizon forms the fundamental plane for this system, i.e., the plane defined by the south and east axes. 
It should be noted that there is a subtle difference between the definition of the impact site's latitude by geodetic or astronomical standards (Vallado, 2013); these become identical by imposing the assumption of a perfectly spherical impacted body (Deimos). The low gravitational acceleration at the surface of Deimos has a negligible effect on the speed of the ejecta, so the assumption of a uniform spherical geometry is justified.

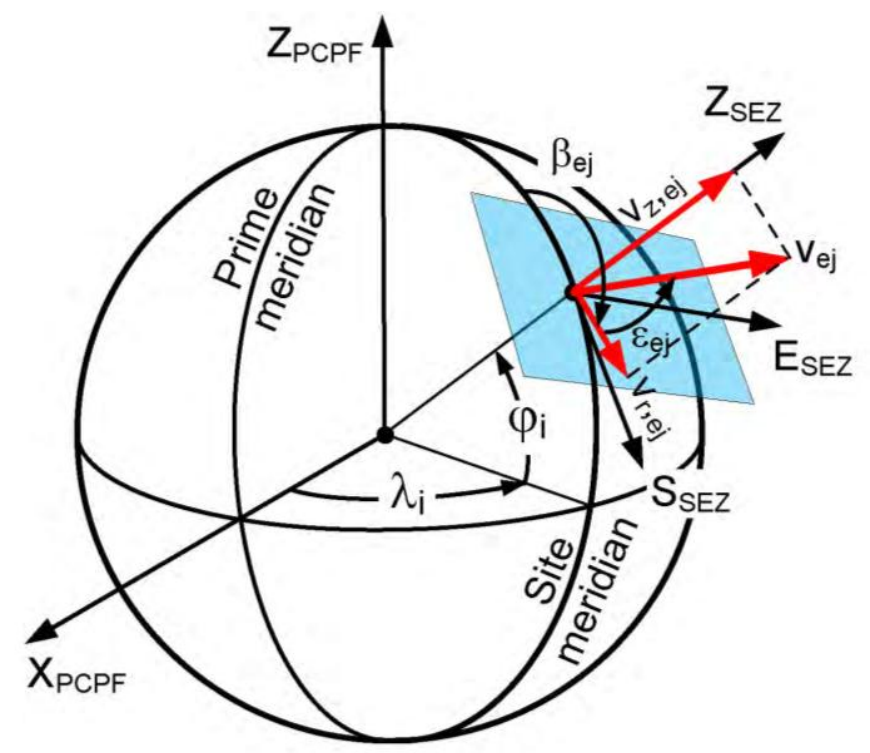

Figure 8. Illustration of the relationship between the Planet-Centered Planet Fixed (PCPF) frame and the South-East-Zenith (SEZ) frames. The fundamental plane is highlighted (blue).

Figure 8 illustrates the relationship between SEZ and PCPF. $\varepsilon_{e j}$ is the elevation angle of the ejection velocity vector from the horizontal, defined as $0 \leq \varepsilon_{e j} \leq 90^{\circ}$. From the Z-model formulation (Equation 1 ), $\varepsilon_{e j}=35.4^{\circ}$. $\beta_{e j}$ is the azimuth of the ejection velocity vector and is measured from the North, positive clockwise as viewed from above the site such that $0 \leq \beta_{e j} \leq 360^{\circ}$, and is sampled at 11 positions across this range separated by $30^{\circ}$. The streamlines defined in Z-model frame can be rotated into SEZ using the relationships:

$$
\begin{gathered}
v_{\text {South }}=v_{r} \cos \left(180^{\circ}-\beta_{e j}\right) \\
v_{\text {East }}=v_{r} \sin \left(180^{\circ}-\beta_{e j}\right)
\end{gathered}
$$

where $v_{r}$ is defined by Equation 2. Subsequently two rotation matrices, the first around the $y$-axis and the second around the z-axis, are required to rotate the SEZ frame into the PCPF frame (specifically, the Voltaire SEZ frame into the DeimosCentered Deimos-Fixed frame). The rotation matrices are:

$$
{ }_{D C D F} R^{S E Z}=\left[\begin{array}{ccc}
\cos \lambda_{i} & -\sin \lambda_{i} & 0 \\
\sin \lambda_{i} & \cos \lambda_{i} & 0 \\
0 & 0 & 1
\end{array}\right]\left[\begin{array}{ccc}
\sin \varphi_{i} & 0 & \cos \varphi_{i} \\
0 & 1 & 0 \\
-\cos \varphi_{i} & 0 & \sin \varphi_{i}
\end{array}\right]
$$


Finally, we can convert the DCDF velocity coordinates to MCI. By manipulating the basic kinematic equation for the position vector of an ejected particle in the DCDF frame, $\vec{r}_{e j}^{D C D F}$, in terms of the known $\vec{v}_{e j}^{D C D F}$ we get the expression:

$$
\vec{v}_{e j}^{M C I}=\frac{d}{d t} \vec{r}_{D}^{M C I}+\vec{v}_{e j}^{D C D F}+{ }^{M C I} \omega^{D C D F} \times \vec{r}_{i}^{D C D F}
$$

$\frac{d}{d t} \vec{r}_{D}^{M C I}=\vec{v}_{D}^{M C I}$, where $\vec{v}_{D}^{M C I}$ is the velocity vector of Deimos in the MCI frame. This is determined from Deimos ephemerides. ${ }^{M C I} \omega^{D C D F}$ is the angular rate of the DCDF frame with respect to MCI; this is the rotation rate of Deimos. $\vec{r}_{i}^{D C D F}$ is the position of the impact site in DCDF coordinates.

Following a similar process, for the impact of ejecta with Phobos the process is reversed to obtain the velocity vector at impact in the Phobos Centered PhobosFixed (PhCPhF) frame. With similar notation as used above this relation is:

$$
\vec{v}_{e j}^{P h C P h F}=\vec{v}_{e j}^{M C I}-\vec{v}_{P h}^{M C I}-{ }^{M C I} \omega^{P h C P h F} \times \vec{r}_{i}^{P h C P h F}
$$

The validity of these equations has been checked with MICE, a commercial level interface created by JPL/Caltech to SPICE ephemeris information from NASA's Navigation and Ancillary Information Facility (naif.jpl.nasa.gov) (Acton et al., 2002).

\section{References}

Acton, C., Backman, N., Elson, L., Semenov, B., Turner, F., Wright, E., 2002. Extending NASA's SPICE ancillary information system to meet future mission needs, in: 2002 AIAA Space Operations Conference Proceedings. pp. 8-11.

Alvarellos, J.L., Zahnle, K.J., Dobrovolskis, A.R., Hamill, P., 2002. Orbital Evolution of Impact Ejecta from Ganymede. Icarus 160, 108-123.

doi:10.1006/icar.2002.6950

Alvarellos, J.L., Zahnle, K.J., Dobrovolskis, A.R., Hamill, P., 2008. Transfer of mass from Io to Europa and beyond due to cometary impacts. Icarus 194, 636-646. doi:10.1016/j.icarus.2007.09.025

Andrews, R.J., 1975. Origin and distribution of ejecta from near-surface laboratoryscale cratering experiments. US Air Force Rep. AFWL-TR-74-314.

Archinal, B.A., A’Hearn, M.F., Bowell, E., Conrad, A., Consolmagno, G.J., Courtin, R., Fukushima, T., Hestroffer, D., Hilton, J.L., Krasinsky, G. a., Neumann, G., Oberst, J., Seidelmann, P.K., Stooke, P., Tholen, D.J., Thomas, P.C., Williams, I.P., 2010. 
Report of the IAU Working Group on Cartographic Coordinates and Rotational Elements: 2009. Celest. Mech. Dyn. Astron. 109, 101-135. doi:10.1007/s10569010-9320-4

Asphaug, E., Collins, G., Jutzi, M., 2015a. Global Scale Impacts, Asteroids IV.

Asphaug, E., Hurford, T., Spitale, J.N., Hemingway, D., Rhoden, A.R., Henning, W.G., Bills, B.G., Walker, M., 2015b. Tidal Disruption of Phobos as Cause of Surface Fractures. Eur. Planet. Sci. Congr. EPSC2015-796.

Asphaug, E., Melosh, H.J., 1993. The Stickney Impact of Phobos: A Dynamical Model. Icarus 101, 144-164.

Avanesov, G., Bonev, B., Kempe, F., 1989. Television observations of Phobos. Nature 341, 585-590.

Barnhart, C.J., Nimmo, F., 2011. Role of impact excavation in distributing clays over Noachian surfaces. J. Geophys. Res. 116, E01009. doi:10.1029/2010JE003629

Basilevsky, A.T., Lorenz, C.A., Shingareva, T.V., Head, J.W., Ramsley, K.R., Zubarev, A.E., 2014. The surface geology and geomorphology of Phobos. Planet. Space Sci. 102, 95-118. doi:10.1016/j.pss.2014.04.013

Bierhaus, E.B., Dones, L., 2014. Craters and ejecta on Pluto and Charon: Anticipated results from the New Horizons flyby. Icarus 246, 165-182. doi:10.1016/j.icarus.2014.05.044

Britt, D.T., Pieters, C.M., 1988. The Origin of PHOBOS-Implication of Compositional Properties. Sol. Syst. Res. 22, 143.

Brown, P., Spalding, R.E., ReVelle, D.O., Tagliaferri, E., Worden, S.P., 2002. The flux of small near-Earth objects colliding with the Earth. Nature 420, 294-296. doi:10.1038/nature01238

Burns, J.A., 1978. The dynamical evolution and origin of the Martian moons. Vistas Astron. 22, 193-210. doi:10.1016/0083-6656(78)90015-6

Burns, J.A., 1972. Dynamical characteristics of Phobos and Deimos. Rev. Geophys. Sp. Phys. 10, 463-483. doi:10.1029/RG010i002p00463

Burns, J.A., Hamilton, D.P., Mignard, F., Soter, S., 1996. The Contamination of Iapetus by Phoebe Dust. Physics, Chem. Dyn. Interplanet. Dust 104.

Cazenave, A., Dobrovolskis, A., Lago, B., 1980. Orbital history of the Martian satellites with inferences on their origin. Icarus 44, 730-744. doi:10.1016/00191035(80)90140-2 
Chappaz, L., Melosh, H.J., Vaquero, M., Howell, K.C., 2011. Transfer of Impact Ejecta Material from the Surface of Mars to Phobos and Deimos, in: AAS/AIAA Spaceflight Mechanics Meeting.

Cintala, M., Berthoud, L., Hörz, F., 1999. Ejection-velocity distributions from impacts into coarse-grained sand. Meteorit. Planet. Sci. 34, 605-623.

Cintala, M.J., Grieve, R.A.F., 1998. Scaling impact-melt and crater dimensions: Implications for the lunar cratering record. Meteorit. Planet. Sci. 33, 889-912.

Curtis, H., 2013. Orbital mechanics for engineering students. ButterworthHeinemann.

Davis, D.R., Greenberg, R., Housen, K.R., 1981. The unusual dynamical environment of Phobos and Deimos. Icarus 47, 220-233. doi:10.1016/0019-1035(81)901688

Dobrovolskis, A.R., Burns, J.A., 1980. Life near the Roche limit - Behavior of ejecta from satellites close to planets. Icarus 42, 422-441. doi:10.1016/00191035(80)90105-0

Farnocchia, D., Chesley, S.R., Chodas, P.W., Tricarico, P., Kelley, M.S.P., Farnham, T.L., 2014. Trajectory analysis for the nucleus and dust of comet C/2013 A1 (Siding Spring) 1, 1-31. doi:10.1088/0004-637X/790/2/114

Gladman, B.J., Burns, J.A., Duncan, M.J., Levison, H.F., 1995. The dynamical evolution of lunar impact ejecta. Icarus. doi:10.1006/icar.1995.1193

Hamelin, M., 2011. Motion of blocks on the surface of Phobos: New constraints for the formation of grooves. Planet. Space Sci. 59, 1293-1307. doi:10.1016/j.pss.2010.05.023

Hermalyn, B., Schultz, P., 2013. New Insights Into the Ejecta Mass-Velocity Distribution: Experimental Time- Resolved Measurements and Applications To Cratering. 44th Lunar Planet. Sci. Conf.

Holsapple, K.A., 1993. The Scaling of Impact Processes in Planetary Sciences. Annu. Rev. Earth Planet. Sci. 21, 333-373.

Horstman, K.C., Melosh, H.J., 1989. Drainage Pits in Cohesionless Materials : Implications for the Surface of Phobos. J. Geophys. Res. 94, 433-441.

Housen, K.R., Holsapple, K.A., 2011. Ejecta from impact craters. Icarus 211, 856-875. doi:10.1016/j.icarus.2010.09.017 
Klacka, J., 2002. On Radiation Pressure and the Poynting-Robertson Effect for Fluffy Dust Particles 361, 349-361. doi:http://dx.doi.org/10.1006/icar.2002.6849

Lambeck, K., 1979. On the orbital evolution of the Martian satellites. J. Geophys. Res. 84, 5651. doi:10.1029/JB084iB10p05651

Lee, P., 2009. First International Conference on the Exploration of Phobos and Deimos: Summary and Recommendations, Mars Institute Technical Publication 2009-001.

Lee, S.W., Thomas, P., Veverka, J., 1986. Phobos, Deimos, and the moon - Size and distribution of crater ejecta blocks. Icarus 86, 77-86. doi:10.1016/00191035(86)90075-8

Leimkuhler, B., Reich, S., 2004. Simulating Hamiltonian Dynamics, Cambridge. ed. Cambridge University Press.

Leinhardt, Z.M., Stewart, S.T., 2012. Collisions Between Gravity-Dominated Bodies. I. Outcome Regimes and Scaling Laws. Astrophys. J. 745, 79. doi:10.1088/0004$637 \mathrm{X} / 745 / 1 / 79$

Love, S.G., Ahrens, T.J., 1996. Catastrophic Impacts on Gravity Dominated Asteroids. Icarus 124, 141-155. doi:10.1006/icar.1996.0195

Lunine, J.I., Neugebauer, G., Jakosky, B.M., 1982. Infrared observations of Phobos and Deimos from Viking. J. Geophys. Res. 87, 10297. doi:10.1029/JB087iB12p10297

Maxwell, D., Seifert, K., 1974. Modeling of cratering, close-in displacements and ejecta. Def. Nucl. Agency, Rep. \#DNA-F-3628.

Maxwell, D.E., 1977. Simple Z model for cratering, ejection, and the overturned flap, in: Impact and Explosion Cratering: Planetary and Terrestrial Implications. pp. 1003-1008.

Melosh, H.J., 1989. Impact cratering: A geologic process 1-240.

Murchie, S., 2010. Mars Reconnaissance Orbiter Spherical Harmonics Binary Data Records, data set MRO-M-RSS-5-SDP-V1. NASA Planet. Data Syst.

Murchie, S., Erard, S., 1996. Spectral Properties and Heterogeneity of Phobos from Measurements by Phobos 2. Icarus 123, 63-86. doi:10.1006/icar.1996.0142

Murchie, S., Erard, S., 1993. The spectrum of PHOBOS from PHOBOS 2 observations at 0.3-2.6 microns: Comparison to previous data and meteorite analogs, in: Lunar and Planetary Science Conference. pp. 1025-1026. 
Murchie, S.L., Britt, D.T., Head, J.W., Pratt, S.F., Fisher, P.C., Zhukov, B.S., Kuzmin, A.A., Ksanfomality, L. V, Zharkov, A. V, Nikitin, G.E., 1991. Color heterogeneity of the surface of Phobos: Relationships to geologic features and comparison to meteorite analogs. J. Geophys. Res. Solid Earth 96, 5925-5945.

Murchie, S.L., Choo, T., Humm, D., Rivkin, A.S., Bibring, J.-P., Langevin, Y., Gondet, B., Roush, T.L., Duxbury, T., Team, C., 2008. MRO/CRISM observations of Phobos and Deimos, in: Lunar and Planetary Science Conference. p. 1434.

Murchie, S.L., Thomas, P.C., Rivkin, A.S., Chabot, N.L., 2003. Phobos and Deimos. Asteroids III 2186-2224.

Murray, E.J.B., Iliffe, J.C., Muller, J.A.L., Neu-, G., Werner, S., Balme, M., Team, H.C., St, G., Universität, F., Building, D., 2006. New Evidence on the Origin of Phobos' Parallel Grooves. Lunar Planet. Sci. XXXVII 1, 3-4.

O'Keefe, J.D., Ahrens, T.J., 1985. Impact and explosion crater ejecta, fragment size, and velocity. Icarus 62, 328-338. doi:10.1016/0019-1035(85)90128-9

Ramsley, K.R., Head, J.W., 2013. Mars impact ejecta in the regolith of phobos: Bulk concentration and distribution. Planet. Space Sci. 87, 115-129. doi:10.1016/j.pss.2013.09.005

Richardson, J.E., Melosh, H.J., Lisse, C.M., Carcich, B., 2007. A ballistics analysis of the Deep Impact ejecta plume: Determining Comet Tempel 1's gravity, mass, and density. Icarus 191, 176-209. doi:10.1016/j.icarus.2007.08.033

Rivkin, A., Brown, R.H., Trilling, D.E., Bell III, J.F., Plasmman, J.H., 2002. Near-Infrared Spectrophotometry of Phobos and Deimos. Icarus 156, 64-75. doi:10.1006/icar.2001.6767

Roddy, D.J., 1977. Large-scale impact and explosion craters-Comparisons of morphological and structural analogs, in: Impact and Explosion Cratering: Planetary and Terrestrial Implications. pp. 185-246.

Rosenblatt, P., Le Maistre, S., Marty, J., Dehant, V., Paetzold, M., Van Hoolst, T., 2008. Improvement of the Mass Determination of Both Martian Moons Using MEX, MGS, ODY and MRO Tracking Data. AGU Fall Meet. 1, 1377.

Schmedemann, N., Michael, G.G., Ivanov, B. a., Murray, J.B., Neukum, G., 2014. The age of Phobos and its largest crater, Stickney. Planet. Space Sci. 102, 152-163. doi:10.1016/j.pss.2014.04.009

Schmidt, R.M., Housen, K.R., 1987. Some recent advances in the scaling of impact and explosion cratering. Int. J. Impact Eng. 5, 543-560. 
Smith, H.D., Lee, P., Hamilton, D., 2015. Low Velocity Impacts on Phobos. 46th Lunar Planet. Sci. Conf. 8477-8478.

Soter, S., 1972. The Dust Belts of Mars. Cornell Univ. Rep. CRSR 462.

Soter, S., 1971. Studies of the Terrestrial Planets. Cornell University.

Stöffler, D., Gault, D.E., Wedekind, J., Polkowski, G., 1975. Experimental hypervelocity impact into quartz sand: Distribution and shock metamorphism of ejecta. J. Geophys. Res. 80, 4062-4077.

Szeto, A.M.K., 1983. Orbital Evolution and Origin of the Martian Satellites. Icarus 133-168.

Thomas, N., Stelter, R., Ivanov, a., Bridges, N.T., Herkenhoff, K.E., McEwen, a. S., 2011. Spectral heterogeneity on Phobos and Deimos: HiRISE observations and comparisons to Mars Pathfinder results. Planet. Space Sci. 59, 1281-1292. doi:10.1016/j.pss.2010.04.018

Thomas, P., 1998. Ejecta Emplacement on the Martian Satellites. Icarus 131, 78-106. doi:10.1006/icar.1997.5858

Thomas, P., 1979. Surface Features of Phobos and Deimos. Icarus 40, 223-243.

Thomas, P., Veverka, J., 1980a. Downslope movement of material on Deimos. Icarus 234-250. doi:10.1016/0019-1035(80)90073-1

Thomas, P., Veverka, J., 1980b. Crater densities on the satellites of Mars. Icarus 41, 365-380. doi:10.1016/0019-1035(80)90221-3

Thomas, P.C., 1993. Gravity, Tides, and Topography on Small Satellites and Asteroids: Application to Surface Features of the Martian Satellites. Icarus 105, 326-344. doi:http://dx.doi.org/10.1006/icar.1993.1130

Thomas, P.C., Adinolfi, D., Helfenstein, P., Simonelli, D., Veverka, J., 1996. The Surface of Deimos : Contribution of Materials and Processes to Its Unique Appearance. Icarus 123, 536-556.

Udrea, B., Nayak, M., Allen, B., Bourke, J., Casariego, G., Gosselin, S., Hiester, E., Maier, M., Melchert, J., Patel, C., 2015. Mars Moons Prospector Mission with CubeSats, in: EGU General Assembly Conference Abstracts. p. 7881.

Udrea, B., Nayak, M. V, Franquiz, F.J., Bourke, J.G., 2016. PIRARUCU: Mars Moons Prospector Mission with Cubesats, in: IEEE Aerospace Conference. Big Sky, Montana. 
Vallado, D.A., 2013. Fundamentals of Astrodynamics and Applications, 4th ed. Microcosm Press.

Veverka, J., 1978. The surfaces of Phobos and Deimos. Vistas Astron. 22, 163-192. doi:10.1016/0083-6656(78)90014-4

Veverka, J., Duxbury, T.C., 1977. Viking observations of Phobos and Deimos: Preliminary results. J. Geophys. Res. 82, 4213-4223. doi:10.1029/JS082i028p04213

Weidenschilling, S.J., 1979. A possible origin for the grooves of Phobos. Nature 282, 697-712.

Wilson, L., Head, J.W., 2015. Groove formation on Phobos: Testing the Stickney ejecta emplacement model for a subset of the groove population. Planet. Space Sci. 105, 26-42. doi:10.1016/j.pss.2014.11.001

Zahnle, K., Alvarellos, J.L., Dobrovolskis, A., Hamill, P., 2008. Secondary and sesquinary craters on Europa. Icarus 194, 660-674.

Zahnle, K., Schenk, P., Levison, H., Dones, L., 2003. Cratering rates in the outer solar system. Icarus 163, 263-289. doi:10.1016/S0019-1035(03)00048-4 